\title{
Separation and purification of amygdalin from thinned bayberry kernels by macroporous adsorption resins \\ Running title: Separation of bayberry amygdalin
}

Tao Wang ${ }^{\mathrm{a}, \mathrm{b}}$, Shengmin $\mathrm{Lu}^{\mathrm{a}}{ }^{\mathrm{k}}$, Qile Xia ${ }^{\mathrm{a}}$, Zhongxiang Fang ${ }^{\mathrm{c}}$, Stuart Johnson ${ }^{\mathrm{c}}$

${ }^{a}$ Key Laboratory of Fruits and Vegetables Postharvest and Processing of Zhejiang

Province, Institute of Food Science, Zhejiang Academy of Agricultural Science,

Hangzhou 310021, PR China

${ }^{b}$ Zhejiang Normal University, Jinhua 321000, PR China

${ }^{c}$ Food Science \& Technology Program, School of Public Health, Faculty of Health

Sciences, International Institute of Agri-Food Security (IIAFS), Curtin University,

GPO Box U1987, Perth, 6845, WA, Australia

${ }^{*}$ Corresponding author at: Key Laboratory of Fruits and Vegetables Postharvest and Processing of Zhejiang Province, Institute of Food Science, Zhejiang Academy of Agricultural Science, Hangzhou 310021, PR China. Tel.: +86 57186417306.

E-mail address: lushengmin@hotmail.com (S. Lu) 


\section{ABSTRACT}

To utilize the low-value thinned bayberry (Myrica rubra Sieb. et Zucc) kernels (TBKs) waste, an efficient method using macroporous adsorption resins (MARs) for separation and purification of amygdalin from TBKs crude extracts was developed. An aqueous crude sample was prepared from a methanol TBK extract, followed by resin separation. A series of MARs were initially screened for adsorption/desorption of amygdalin in the extract, and D101 was selected for characterization and method development. The static adsorption data of amygdalin on D101 was best fitted to the pseudo-second-order kinetics model. The solute affinity towards D101 at $30{ }^{\circ} \mathrm{C}$ was described and the equilibrium experimental data were well-fitted to Langmuir and Freundlich isotherms. Through one cycle of dynamic adsorption/desorption, the purity of amygdalin in the extract, determined by HPLC, increased about 17-fold from $4.8 \%$ to $82.0 \%$, with $77.9 \%$ recovery. The results suggested that D101 resin effectively separate amygdalin from TBKs.

Keywords: Bayberry; Amygdalin; Tthinned bayberry kernels (TBKs); Macroporous adsorption resins (MARs); Purification 


\section{Introduction}

Bayberry (Myrica rubra Sieb. et Zucc.) is a fruit tree native to China, mainly south of Yangtze river, and typically in Zhejiang province. The bayberry tree has a high fruit-setting rate and manual thinning (removal of young, immature fruits during the growing season) is commonly practiced to achieve a higher productivity and improve quality of the remaining fruit [1]. The thinned fruits are usually regarded as waste and discarded. Compared with the mature bayberry fruit, thinned fruit has a higher proportion of stones in a range of $19.6-34.2 \%(\mathrm{~g} / 100 \mathrm{~g}$ fresh weight $[\mathrm{FW}])$. Each fruit stone contains one kernel and these kernels from mature fruit stone are reported to be rich in nutrients such as crude fiber, fat, vitamins, proteins and minerals [2]. The kernels may be crushed and used as nutritious supplements for animal feed and also have potential for development into high value-added products such as polyphenols, vegetable fats and vegetable protein [3]. Preliminary investigations on TBKs reported average contents of moisture, protein, fats and carbohydrates of $30.2 \%$, $28.0 \%, 58.4 \%$ and $1.8 \%$ respectively. In addition to valuable nutritional components, bayberry kernels have also been reported to contain amygdalin, a cyanogenic glycoside with potential pharmacological and toxicological effects [2].

Amygdalin is found in seeds and leaves of loquat, apricot, peach, plum and other Rosaceae species. Amygdalin is an $\alpha$-hydroxy benzyl nitrile, consisting of the aglycone mandelonitrile, and the disaccharide gentiobiose (6-o- $\beta$-d-glucopyranosyl-d-glucose) (Fig.1), with a formula of $\mathrm{C}_{20} \mathrm{H}_{27} \mathrm{O}_{11} \mathrm{~N}$ and 
molecular weight of 458 [4]. It was reported that amygdalin may play a role in relieving cough and asthma in humans [5]; regulate immune function and demonstrate anti-tumor activity [6]. Cyanogenic glycosides have high toxicity potential upon ingestion, however the toxicity potential for amygdalin was reported as very low [7]. Amygdalin from loquat seeds has been proved to be therapeutically potential in preventing and / or treating of atherosclerosis, gastric ulcer, psoriasis, arthritis and wound healing [8-12]. However there is little reported research on amygdalin from TBKs, and its phytochemical properties and possible medicinal uses are not fully investigated.

Macroporous adsorption resins (MARs) are efficient matrices for enrichment of bioactive substances from plant resources due to their high selectivity and adsorption capacity, as well as stability and resistance to degradation by osmotic shock and oxidation [13]. They are a group of polymers containing a permanent network of pores independent of the state of swelling of the resin and thus display much better solvent tolerance than gel-type resins. The adsorption performance of MARs is closely related to its polarity. Non-polar resins with strong hydrophobic pore surface, without any functional groups, are suitable for adsorbing non-polar substances; medium-polar resins with both hydrophilic and hydrophobic surface properties, containing an ester group, are suitable for adsorbing both non-polar and polar substances; and polar resins adsorb polar substance mainly through electrostatic interactions [14-15]. The isolation of plant polyphenolic compounds routinely use MARs such as Amberlite XAD resin or Sephadex [16-18]. Although solid-phase 
extraction and purification (e.g. $\mathrm{C}_{18}$ Sep-Pak cartridge) are commonly used before HPLC determination of bioactive compounds [19], MARs are more efficient in enrichment of bioactive compounds if its concentration in plant is relatively low [13]. To our knowledge, there is no published report using MARs for purification of cyanogenic glycosides.

An efficient method for the purification of amygdalin from TBKs might provide value-addition opportunities for the utilization of this low-value thinned fruit waste. Therefore the aim of this study was to determine the static and dynamic adsorption/desorption parameters of amygdalin from TBKs extract using macroporous resin chromatography.

\section{Materials and methods}

\subsection{Chemicals, reagents and samples}

Ethanol of analytical grade was purchased from East Pharmaceutical Technology Co., Ltd. (Hangzhou, China); Acetonitrile of HPLC grade, and amygdalin were purchased from J\&K Scientific Ltd. (Beijing, China). Distilled water was used throughout. The cultivar of thinned bayberry fruits was Ding-ao which was collected from Wenzhou city (Zhejiang Province, China) in June, 2012. When the thinned bayberry was arrived at our laboratory, stones were separated by removing the outer villiform pulp after lyophilizing the whole fruits. The stones were broken by cracking 
102

103

104

with a micro-punch to obtain the kernels $(5.68 \pm 0.33 \mathrm{~g}$ of weight/100 kernels in average), and the kernels were sealed in aluminum foil bags and stored at $-40^{\circ} \mathrm{C}$ for subsequent analysis.

MARs of HPD722, HPD720, AB-8, NKA-9, D101, HPD100 and HPD400 were purchased from Cangzhou Bon Adsorber Technology Co., Ltd. (Hebei Province, China). These are the most commonly used resins in separation of plant bioactive substance in China [20-21]. Specifications of the MARs are shown in Table 1. The resins were soaked in $95 \%$ ethanol for $24 \mathrm{~h}$ and then washed by distilled water before use.

\subsection{HPLC analysis of amygdalin}

The HPLC system (Dalian Elite Instrument Co., Ltd., China) consisted of a P230 pump equipped with a $230^{+}$diode array detector set at $214 \mathrm{~nm}$. A Symmetry® C18 MG column ( $4.6 \mathrm{~mm} \times 250 \mathrm{~mm}, 5 \mu \mathrm{m}$, Waters Ltd., Ireland) was maintained at $25^{\circ} \mathrm{C}$. The mobile phase was $13 \%$ acetonitrile in water at a flow rate of $1.0 \mathrm{ml} / \mathrm{min}$. Standard solutions of amygdalin with the concentrations of $0,100,200,300,400 \mathrm{mg} \mathrm{l}^{-1}$ were filtered through $0.45 \mu \mathrm{m}$ membrane filters and each of $10 \mu \mathrm{l}$ was injected into the HPLC system to get the standard curve. The concentration of amygdalin both in crude and purified extracts was determined as follows [22]: $0.25 \mathrm{~g}$ extract was dissolved in $25 \mathrm{ml}$ methanol solution, treated in ultrasound $(250 \mathrm{~W}, 50 \mathrm{kHz})$ for $30 \mathrm{~min}$; and then

filtered through a Whatman No. 1 filter paper using a Buchner funnel. A $1 \mathrm{ml}$ extract 
solution was diluted 50x with 50\% methanol (aq.) and injected into the HPLC system and its concentration was determined from the calibration curve.

\subsection{Preparation of sample solutions of amygdalin crude extract}

The bayberry kernels were dried at $105^{\circ} \mathrm{C}$ for enzyme inactivation and defatted with 20 volumes petroleum ether (boiling at $60-90^{\circ} \mathrm{C}$ for $2 \mathrm{~h}$ ) for three times. Then the kernels were ground into powder and passed through a 60 mesh sieve. Bayberry kernel powder was extracted with 20 volumes methanol solution in a flask $\left(45^{\circ} \mathrm{C}\right.$ for $30 \mathrm{~min}$ ), and repeated three times. The extract solutions were combined and vacuum filtered through a Whatman No. 1 filter paper using a Buchner funnel. The filtrate was evaporated in a rotary evaporator (RE 52-99, Shanghai Yarong Biochemical Instrument Factory, China) under reduced pressure at $45^{\circ} \mathrm{C}$ to remove the methanol. The residue was diluted with distilled water to give the crude TBK solution before being loaded onto the chromatography column.

\subsection{Static adsorption/desorption experiments}

\subsubsection{Adsorption resin screening}

The amygdalin static adsorption experiments using different resins were carried out as follows: $0.5 \mathrm{~g}$ resin was added into $50 \mathrm{ml}$ crude TBK solution with an amygdalin concentration of $380.76 \mathrm{mg} \mathrm{l}^{-1}$ (determined by HPLC) in a $250 \mathrm{ml}$ conical 
flask. The flask was continually shaken in a HZ-9511K incubator (100 rpm) (Hua Lida Experimental Equipment Co., Ltd., Jiangsu province, China) at $30{ }^{\circ} \mathrm{C}$ for $24 \mathrm{~h}$. After the adsorption equilibrium, the sample was filtered through a $0.45 \mu \mathrm{m}$ membrane filter and analyzed by HPLC as in section 2.2. The static desorption experiments were carried out as follows: $60 \mathrm{ml}$ ethanol (aq.) at a concentration of 50\% $(v / v)$ was added to the adsorbate-laden resins separated from the adsorption solution in the $250 \mathrm{ml}$ conical flask. The flask was continually shaken in the incubator (100 rpm) at $30^{\circ} \mathrm{C}$ for $24 \mathrm{~h}$. After filtration through a Whatman No. 1 filter paper using a Buchner funnel, the concentration of amygdalin in desorption solution was analyzed by HPLC. The optimum resin of MARs was determined by their efficiency and capacities of adsorption/desorption, and the calculation equations were as follows:

Adsorption efficiency:

$$
A(\%)=\frac{C_{0}-C_{e}}{C_{0}} \times 100 \%
$$

Adsorption capacity:

$$
q_{e}=\frac{V_{0} \times\left(C_{0}-C_{e}\right)}{W \times(1-M)}
$$

Desorption efficiency:

$$
D(\%)=\frac{C_{d} \times V_{d}}{\left(C_{0}-C_{e}\right) \times V_{0}} \times 100 \%
$$

Where, $A$ is the adsorption efficiency (\%), $q_{e}$ is the adsorption capacity (mg g ${ }^{-1}$ dry resin) at adsorption equilibrium, and $D$ is the desorption efficiency (\%). $C_{0}$ and $C_{e}$ are the initial and equilibrium concentrations of amygdalin solutions, respectively ( $\left.\mathrm{mg} \mathrm{l}^{-1}\right)$, and $C_{d}$ is the amygdalin concentration in the desorption solution $\left(\mathrm{mg} \mathrm{l}^{-1}\right) . W$ is the 
167

weight of the resin (g); $M$ is the moisture content of resin (\%). $V_{0}$ and $V_{d}$ are the volume of amygdalin solution and ethanol (aq.) respectively (ml).

\subsubsection{Adsorption kinetics}

Static adsorption kinetics curves describe the change of adsorption amount over time. The adsorption kinetics curve of amygdalin on the selected resin was studied as following process: $0.5 \mathrm{~g}$ selected resin was added into $50 \mathrm{ml}$ amygdalin solution (114.71 $\mathrm{mg} \mathrm{l}^{-1}$ ) in a $250 \mathrm{ml}$ conical flask. The flask was continually shaken (100 rpm) in an incubator at $30{ }^{\circ} \mathrm{C}$ for $24 \mathrm{~h}$. Liquid sample of $0.5 \mathrm{ml}$ was pipetted at time intervals of $5,10,15,30,60,120$ and $180 \mathrm{~min}$, and the concentration of amygdalin was determined by HPLC. Triplicates were performed for this analysis.

To better understand the adsorption mechanism, the pseudo-first-order, pseudo-second-order and intra-particle diffusion kinetic model were used to evaluate the adsorption kinetics of amygdalin to the selected resins. These models are commonly used in adsorption kinetics study and the model equations are as follows [23].

Pseudo-first-order model:

$$
\log \left(q_{e}-q_{t}\right)=-\frac{k_{1}}{2.303} t+\log q_{e}
$$

Pseudo-second-order model:

$$
\frac{t}{q_{t}}=\frac{1}{q_{e}} t+\frac{1}{k_{2} q_{e}{ }^{2}}
$$

Intra-particle diffusion kinetic model:

$$
q_{t}=k_{i d} t^{1 / 2}+C
$$


191 pseudo-first-order model, pseudo-second-order model and intra-particle diffusion

192 kinetic model, respectively; and $C\left(\mathrm{mg} \mathrm{g}^{-1}\right)$ is a constant, representing boundary layer 193 thickness.

\subsubsection{Adsorption isotherm}

Adsorption isotherms describe the equilibrium distribution of a solute between the

197 adsorbent and the liquid phase. In practice, Langmuir model and Freundlich model are considered to be the most popular models for adsorption process [24]. The Langmuir model assumes monomolecular layer adsorption with a homogeneous distribution of adsorption energies and without mutual interaction between adsorbed molecules [25]. The Freundlich model is a two-parameter model for liquid and gas phase adsorption [26].

Six crude TBK solutions with different initial amygdalin concentrations (26.05, $77.75,129.13,275.04,358.68$ and $728.35 \mathrm{mg} \mathrm{l}^{-1}$ ) were employed to investigate the effect of amygdalin concentration on the adsorption process. The selected resin $(0.5 \mathrm{~g})$ was added to each crude TBK solution $(50 \mathrm{ml})$ and shaken $(100 \mathrm{rpm})$ for $24 \mathrm{~h}$ at $30{ }^{\circ} \mathrm{C}$. The initial and equilibrium concentrations of amygdalin were determined by HPLC to obtain the adsorption isotherm of amygdalin on the resin. The Langmuir and Freundlich models were used to interpret the adsorption experimental data, and the theoretical model parameters values were obtained from the linearized equations at 
$30{ }^{\circ} \mathrm{C}$. The equations are as follows:

Langmuir model:

$$
\mathrm{q}_{\mathrm{e}}=\frac{q_{m} C_{e}}{K_{L}+C_{e}}
$$

$$
\text { Freundlich model: }
$$

$$
\mathrm{q}_{\mathrm{e}}=K_{F} C_{e} e^{\frac{1}{n}}
$$

where, $q_{m}\left(\mathrm{mg} \mathrm{g}^{-1}\right)$ is the maximum adsorption capacity to form monolayer on the resin. $K_{L}$ is the parameter relative to the adsorption energy. $K_{F}$ and $n$ reflect the adsorption capacity and adsorption intensity of the resin respectively. $q_{e}\left(\mathrm{mg} \mathrm{g}^{-1}\right)$ and $C_{e}\left(\mathrm{mg} \mathrm{l}^{-1}\right)$ are the same in equation (2).

\subsubsection{Optimization of the eluent concentration}

After the adsorption equilibrium, the resin was filtered and washed with pure water. Afterwards, $0.5 \mathrm{~g}$ adsorbate-laden resin and $60 \mathrm{ml}$ ethanol eluent at different concentrations in a range of $20-90 \%(\mathrm{v} / \mathrm{v})$ were added into each of the $250 \mathrm{ml}$ conical flasks and shaken $(100 \mathrm{rpm})$ for $24 \mathrm{~h}$ at $30^{\circ} \mathrm{C}$. The amygdalin concentrations in desorption solutions were determined by HPLC. Desorption efficiency of amygdalin to each elute concentration was obtained by calculation as in equation (3).

\subsection{Dynamic adsorption/desorption experiments}

Dynamic adsorption/desorption experiments were carried out in a glass column $(2.5 \times 30 \mathrm{~cm})$ packed with the selected resin $(\sim 24 \mathrm{~g}$ in dry weight $)$. The bed volume 
233 (BV) and the length of the wet-packed resin were $60 \mathrm{ml}$ and $23 \mathrm{~cm}$, respectively. The 234 adsorption/desorption process was performed as follows: $64 \mathrm{ml}$ crude TBK solution 235 with the concentration of $302.72 \mathrm{mg} \mathrm{l}^{-1}$ was loaded onto the column, and the column 236 was firstly washed with distilled water, and subsequently with ethanol solution. The 237 fraction collector was used for collecting the eluent solution. About $8 \mathrm{ml}$ eluent was 238 collected in each tube and every five tubes were combined to determine the concentration of amygdalin by HPLC. The change of amygdalin concentration with eluent volume was plotted to get the dynamic desorption curve. In addition, the eluent solutions were further concentrated and dried under vacuum to calculate the purity of the product. loading volume experiment was performed as follows: $200 \mathrm{ml}$ crude TBK solution column, then the effluent solution was collected and its amygdalin concentration was monitored. The change of amygdalin concentration in the effluent with the loading volume was plotted to get the dynamic leakage curve and the leakage point. The leakage point was defined at which effluent solution concentration reached $10 \%$ of the initial concentration of loading solution [27]. 
Chicago, IL) was used for the determination of differences from triplicates

257

determinations. The results were expressed as means \pm standard deviations and considered significantly different when $P<0.05$.

\section{Results and discussion}

\subsection{Standard curve of amygdalin and the concentration in the crude extract}

According to the method in section 2.2, a very good regression equation for HPLC determination of amygdalin $\left(y=4.7657 x+8.562, \mathrm{R}^{2}=0.9997\right)$ was obtained, where $y$ was the peak area of amygdalin and $x$ was the amygdalin concentration (mg

$1^{-1}$ ). The HPLC chromatograms of the crude TBK sample and authentic amygdalin standard are shown in Fig.2. The purity of amygdalin in the crude TBK sample was determined to be $4.83 \%(\mathrm{w} / \mathrm{w})$.

\subsection{Screening of optimum resin}

Adsorption/desorption properties of seven MARs from the static adsorption/desorption tests are shown in Fig. 3(A). For adsorption efficiency, resins of HPD722, HPD720, AB-8, NKA-9, and D101 were higher than the other two resins of HPD100, HPD400. However, for desorption efficiency, HPD720, AB-8 and D101 
resins were significantly higher than those of other resins. Thus, HPD720, AB-8 and D101 were selected for further screening. Crude TBK solutions with different concentrations were mixed with one of the three kinds of resins, the next steps were done as in section 2.4.1, and then the adsorption capacity and desorption efficiency were obtained, as shown in Fig. 3(B). Resins of D101 and AB-8 showed similar adsorption capacity and were higher than that of HPD720 resin. However, the desorption efficiency of D101 was considerably higher than those of the other two resins.

It was suggested that the performance of MARs is related to its physical and chemical properties, and the efficiency of a chromatographic process is mainly dependent on the adsorbent, the structure and polarity of resins [28]. Through small dipole moment, non-polar macroporous resins are polymerized by monomers which have strong hydrophobicity on pore surface albeit without any functional groups. Therefore, non-polar macroporous resins could effectively interact with the hydrophobic moiety of amygdalin. In the present study, D101 resin showed an outstanding adsorption/desorption properties with amygdalin due to its excellent non-polarity (Table 1). Considering the adsorption/desorption efficiency and adsorption capacity, D101 was selected as the resin for isolating of amygdalin in the TBK sample in further investigations.

\subsection{Adsorption kinetics of amygdalin on D101 resin}


Fig.3(C). The adsorption rate of D101 for amygdalin rapidly increased in the first 25 min, and thereafter increased slowly. The adsorption reached equilibrium at about 45 min. The rapid adsorption process of D101 in the first 25 min was probably a result of the high diffusivity of amygdalin into the porous structure of the resin. The adsorption rate decreased as amygdalin concentration decreased as well as when the resin binding surface is approaching saturation. pseudo-first-order equation, pseudo-second-order equation and intra-particle diffusion kinetic equation were $0.5974,0.9999$ and 0.4724 , respectively. Thus the pseudo-second-order was considered to be the most suitable model for describing the concentrations of both adsorbate and adsorbent were involved in rate determining step

\section{9]. Although the plot for the intra-particle diffusion kinetic model $\left(R^{2}=0.4724\right)$} showed low representation of the whole adsorption process, it could explain the mechanism of adsorption in particular stages. In the present study, the adsorption process of amygdalin included the boundary layer diffusion stage $(0-5 \mathrm{~min})$, the gradual adsorption stage (5-25 min) which was intra-particle diffusion rate-limited, and the equilibrium stage (25-45 min) (Fig. 3(C)). These stages were also observed in separation of phenolics and rosmarinic acid from Rabdosia serra using macroporous resins [30]. Therefore, multiple rate-controlled steps had mutual effects on the 
adsorption process of amygdalin on resin D101.

\subsection{Adsorption isotherm of amygdalin on D101}

To further investigate the adsorption behavior of amygdalin on D101 resin, the equilibrium adsorption isotherm was constructed at the temperature of $30{ }^{\circ} \mathrm{C}$. The initial amygdalin concentration of crude TBK solution was 26.05, 77.75, 129.13, 275.04, 358.68 and $728.35 \mathrm{mg} \mathrm{l}^{-1}$, respectively. As shown in Fig. 3(D), the equilibrium adsorption isotherm of amygdalin on D101 resin tended to be a straight line which suggested that the adsorption process occurred in a very dilute solution [24]. The adsorption rate of D101 increased with the amygdalin concentration of crude TBK solution, considerable in the range of 129.13-358.68 $\mathrm{mg} \mathrm{l}^{-1}$, and reached a saturation plateau when the amygdalin concentration was $358.68 \mathrm{mg} \mathrm{l}^{-1}$. Thus, the amygdalin concentration of crude TBK solution for adsorption was selected between the ranges of 129-358 $\mathrm{mg} \mathrm{l}^{-1}$.

The Langmuir Eq. (7) was converted to the linearized form with $C_{e}$ and $C_{e} / q_{e}$ as independent variable, and the Freundlich Eq. (8) was converted to the power form with $C_{e}$ and $q_{e}$ as independent variable. The experimental data were statistically analyzed, and the parameters $K_{L}, K_{F}$ and $n$ was obtained simultaneously. As shown in Table 2, the correlation coefficients of both Langmuir and Freundlich equations for amygdalin were rather high, and this indicated the adsorption process was a monomolecular layer adsorption with a homogenous distribution among the 
343

adsorption sites at different energies [20]. In most cases, when the parameter $n$ in the Freundlich model is between 1 and 10, the resin shows beneficial adsorption [31]. In the present study the $\mathrm{n}$ value was 1.1062 , which indicated that D101 resin is appropriate for the separation of amygdalin.

\subsection{Optimization of the eluent concentration}

Since amygdalin is mainly used as a pharmaceutical ingredient, aqueous ethanol which is low in toxicity, was selected as the eluent. First, the eluent rate was selected as 1,2 and $3 \mathrm{BV} / \mathrm{h}$, and the desorption efficiency was $82.66,80.88$ and $76.99 \%$, respectively. With the view to shorter working time and lower volume consumption, the desorption flow rate was set as $2 \mathrm{BV} / \mathrm{h}$.

As shown in Fig. 3(E), the concentration of ethanol (aq.) had a significant impact $(P<0.05)$ on the desorption efficiency of amygdalin. When the ethanol concentration was $20 \%$ and $30 \%$, the desorption efficiency were rather low (16.33 and $22.14 \%$ respectively). With the ethanol concentration increased, the desorption efficiency was significantly increased, and reached the maximum of $80.79 \%$ at $40 \%$ ethanol (aq.) $(v / v)$. The proposed mechanism of the elution process was that the eluent (ethanol aq.) outcompeted the adsorbate for adsorption onto the macroporous resin and this led to partial dissolution of the adsorbate into eluent [32]. In the present study, as the proportion of ethanol increased, the competitiveness of which was enhanced and the dissolution rate of amygdalin also improved. As the ethanol concentration increased 
further, the desorption efficiency decreased, possibly due to increasing concentration of alcohol-soluble impurities in the eluent and resulting in the decreasing of amygdalin concentration. Thus, the optimal eluent for desorption of amygdalin from D101 resin was $40 \%$ ethanol (aq.) $(v / v)$.

\subsection{Dynamic adsorption / desorption of amygdalin on D101 resin}

The dynamic adsorption/desorption experiment was performed by the column chromatography with the D101 resin. The character of Langmuir type isotherm implies that with the adsorption sites of adsorbent being covered, it is more and more difficult for the adsorbate molecules to collide with adsorption sites, i.e., the adsorbate molecules have no strong competition with the solvent molecules on the adsorbent surface, and the adsorption finally reaches the equilibrium [24]. In order to evaluate the relationship between the mass of resin and loading volume of TBK crude solutions, the dynamic adsorption of amygdalin on D101 resin was investigated. According to the definition of leakage point, results in Fig.3 (F) indicated that when the loading volume was $64 \mathrm{ml}$, the concentration of amygdalin in effluent solution was $19.21 \mathrm{mg}$ $\mathrm{l}^{-1}$, about $10 \%$ of the initial amygdalin concentration $\left(207.25 \mathrm{mg} \mathrm{l}^{-1}\right)$. Therefore, the most suitable loading volume of TBKs crude solution was $64 \mathrm{ml}$ (about $1 \mathrm{BV}$ ). In addition, the adsorption efficiency at three loading flow rate $(1,2$ and $3 \mathrm{BV} / \mathrm{h})$ were $87.84,91.00$ and $86.24 \%$, respectively, which indicated the loading flow rate to give the highest adsorption efficiency was $2 \mathrm{BV} / \mathrm{h}$. 

initial amygdalin concentration of $302.72 \mathrm{mg} \mathrm{l}^{-1}$ was loaded onto a D101 resin packed glass column, and then washed by $3 \mathrm{BV}$ distilled water to remove the non-adsorbed impurities such as proteins, polysaccharides, etc. The dynamic desorption curve of amygdalin eluted by ethanol (aq.) $(40: 60, v / v)$ was then obtained. As described in Fig.3 (G), about $650 \mathrm{ml}$ (equivalent to $11 \mathrm{BV}$ ) of ethanol eluted the majority of the adsorbed amygdalin. The purity of amygdalin in the lyophilized eluent, as measured by HPLC (Fig. 4), was $82.0 \%$, and the recovery of the amygdalin was $77.9 \%$.

\section{Conclusions}

This study developed a method for separation and purification of amygdalin from TBKs. As a result of $82.0 \%$ purity and $77.9 \%$ recovery of amygdalin in the enriched product, D101 macroporous resin chromatography with the elution by $40 \%$ ethanol (aq.) $(v / v)$ at $30^{\circ} \mathrm{C}$ was found suitable for the separation of amygdalin from the crude extracts with $4.8 \%$ purity. The results evidenced a good adsorption and separation potential of the D101 resin to amygdalin.

\section{Conflict of interest}

The authors declare that there are no conflicts of interest. 


\section{Acknowledgements}

Leading Team of Sci. \& Technol. Innovation (2010R50032).

\section{References:}

416

417

418

419

420

421

422

423

424

425

426

427

428

429

430

[1] K.S. Chen, C.J. Xu, B. Zhang, I.B. Ferguson, Hortic. Rev. 30 (2004) 83.

[2] J. Cheng, X. Ye, J. Chen, D. Liu, S. Zhou, Food Chem. 107 (2008) 1674.

[3] Y. Zhang, S. Li, C. Yin, D. Jiang, F. Yan, T. Xu, Food Chem. 135 (2012) 304.

[4] J.Y. Koo, E.Y. Hwang, S. Cho, J.H. Lee, Y.M. Lee, S.P. Hong, J. Chromatogr. B 814 (2005) 69.

[5] J. Mu, J. Chin. Med. Mat. 25 (2002) 366.

[6] C. Zhou, L. Qian, H. Ma, X. Yu, Y. Zhang, W. Qu, X .Zhang, W. Xia, Carbohydr. Polym. 90 (2012) 516.

[7] J.Vetter. Toxicon, 38 (2000) 11.

[8] J. Deng, C. Li, H. Wang, E. Hao, Z. Du, C. Bao, J. Lv, Y. Wang, Biochem. Biophys. Res. Commun. 411 (2011) 523.

[9] H. Hwang, P. Kim, C. Kim, H. Lee, I. Shim, C. Yin, Y. Yang, D. Hahm, Biol. Pharm. Bull. 31 (2008) 1559.

[10] H. Hwang, H. Lee, C. Kim, I. Shim, D. Hahm, J. Microbiol. Biotechnol. 18 (2008) 1641. 
431

432

433

434

435

436

437

438

439

440

441

442

443

444

445

446

447

448

449

450

451

452

[11] G.A. Fernandez, A.C. Lagunas, S.A. Llebaria, J.J. Perez, Europe Patent EP 1847270 B1 (2010).

[12] F. Nabavizadeh, A.M. Alizadeh, Z. Sadroleslami, S. Adeli, J. Med. Plants Res. 5 (2011) 3122 .

[13] J. Li, H.A. Chase, Nat. Prod. Rep. 27 (2010) 1493.

[14] Y.J. Fu, Y.G.. Zu, W. Liu, C.L. Hou, L.Y. Chen, S.M. Li, X.G. Shi, M.H. Tong, J. Chromatogr. A 2(2007) 206.

[15] Q.P. Xiong, Q.H. Zhang, D.Y. Zhang, Y.Y. Shi, C.X. Jiang, X.J. Shi, Food Chem. 145 (2014) 1.

[16] F. Lalaguna, J. Chromatogr. A 657 (1993) 445.

[17] C. Datta, A. Dutta, D. Dutta, S. Chaudhuri, Procedia Food Sci. 1(2011) 893.

[18] A. Bunea, D. Rugină, Z. Sconţa, R. M. Pop, A. Pintea, C. Socaciu, J. VanCamp, Phytochem. 95 (2013) 436.

[19] A. A. García, B. C. Grande, J. S. Gándara, J Chromatogr. A. 1054 (2004) 175.

[20]W. Liu, S. Zhang, Y. Zu, Y. Fu, W. Ma, D. Zhang, Y. Kong, X. Li, Bioresour. Technol., 101 (2010) 4667.

[21] Y. Sun, H. Yuan, L. Hao, C. Min, J. Cai, J. Liu, P. Cai, S. Yang, Food Chem. 141 (2013) 533.

[22] National Pharmacopoeia Committee, Pharmacopoeia of the People's Republic of China (Part 1), Chemical Industry Press, Beijing, China (2010) pp.260

[23] Y.S. Ho, G. McKay, Chem. Eng. J. 70 (1998) 115.

[24] K. Seiichi, T. Ishikawa, I. Abe, Adsorption Science, Chemical Industry Press, 
[26] H. Freundlich, Z. Phys. Chem. 57 (1907) 385.

[27] J. Li, H.A. Chase, J. Chromatogr. A 1216 (2009) 8759.

457

[28] C. Nobrea, M.J. Santos, A. Dominguez, D. Torresa, O. Rocha, A.M. Peres, I. Rocha, E.C. Ferreira, J.A. Teixeira, L.R. Rodrigues, Anal. Chim. Acta 654 (2009)

459 71.

460 [29] R. Wang, X. Peng, L. Wang, B. Tan, Y. Liu, Y. Feng, J. Sep. Sci., 35 (2012).1985.

461 [30] L. Lin, H. Zhao, Y. Dong, B. Yang, M. Zhao, Food Chem.130 (2012) 417.

462

[31] G. Annadurai, S. Rajesh, K.P.O. Babu, T. Mahesh, T. Murugesan, Bioprocess Biosys. Eng. 22 (2000) 493.

464

[32] P.R. Brown, E. Grushka, Adv. Chromatogr. 42 (2001) 25.

465 
Fig.1 Chemical structure of amygdalin

Fig.2 HPLC chromatograms of authentic amygdalin standard (A) and crude TBK sample (B)

Fig.3 Adsorption/desorption behaviors of amygdalin from TBKs extract on MARs. (A) Adsorption/desorption ratios of amygdalin on different resins (the number in $\mathrm{X}$-axis represented different types of resins: 1.HPD722, 2.HPD720, 3.AB-8, 4.NKA-9, 5.D101, 6.HPD100, and 7.HPD400). Different lowercase letters above each bar represented significance difference at $P<0.05$. Experiments were done in triplicate; (B) Adsorption capacity and desorption ratio of amygdalin on three types of resins and three amygdalin concentrations at $30^{\circ} \mathrm{C}$; (C) Static adsorption kinetics of amygdalin on D101 resin; (D) Adsorption isotherm of amygdalin on D101 resin at $30{ }^{\circ} \mathrm{C}$. Point A was the equilibrium concentration $\left(C_{e}\right)$ of amygdalin solution corresponding to the adsorption capacity when the initial concentration was $129.13 \mathrm{mg} / \mathrm{L}$, and Point B was that of $358.68 \mathrm{mg} / \mathrm{L}$; (E) Effect of ethanol concentration on desorption ratio of amygdalin on D101 resin. Different lowercase letters above each bar represented significance difference at $P<0.05$. Experiments were done in triplicate; (F) Dynamic adsorption curve of amygdalin on D101 resin; and (G) Dynamic desorption curve of amygdalin on D101 resin.

Fig.4 The chromatogram of enriched amygdalin after D101 resin purification 
Fig.1 T. Wang, et al.

486
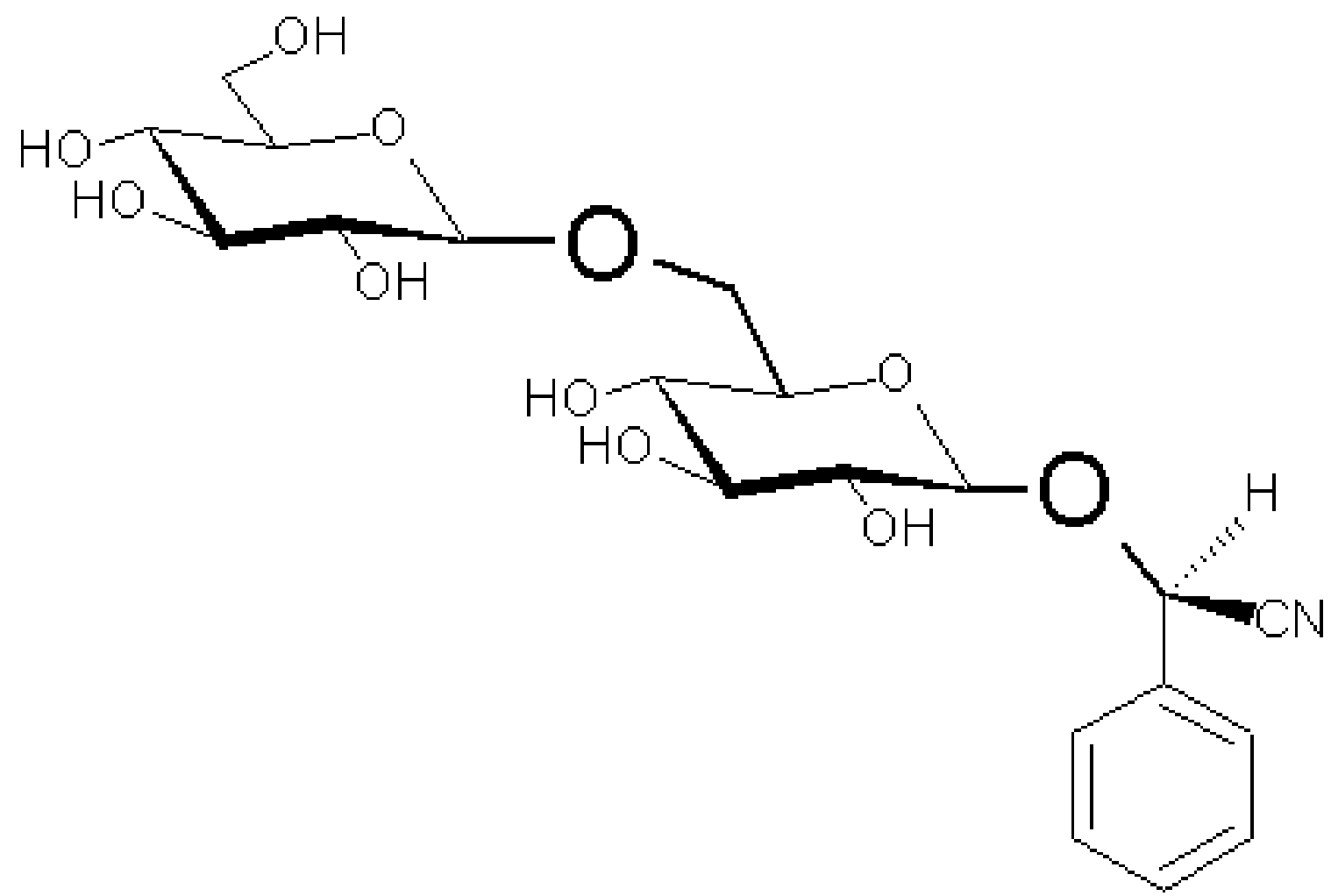

487

488 
Fig.2 (A) and (B) T. Wang, et al.
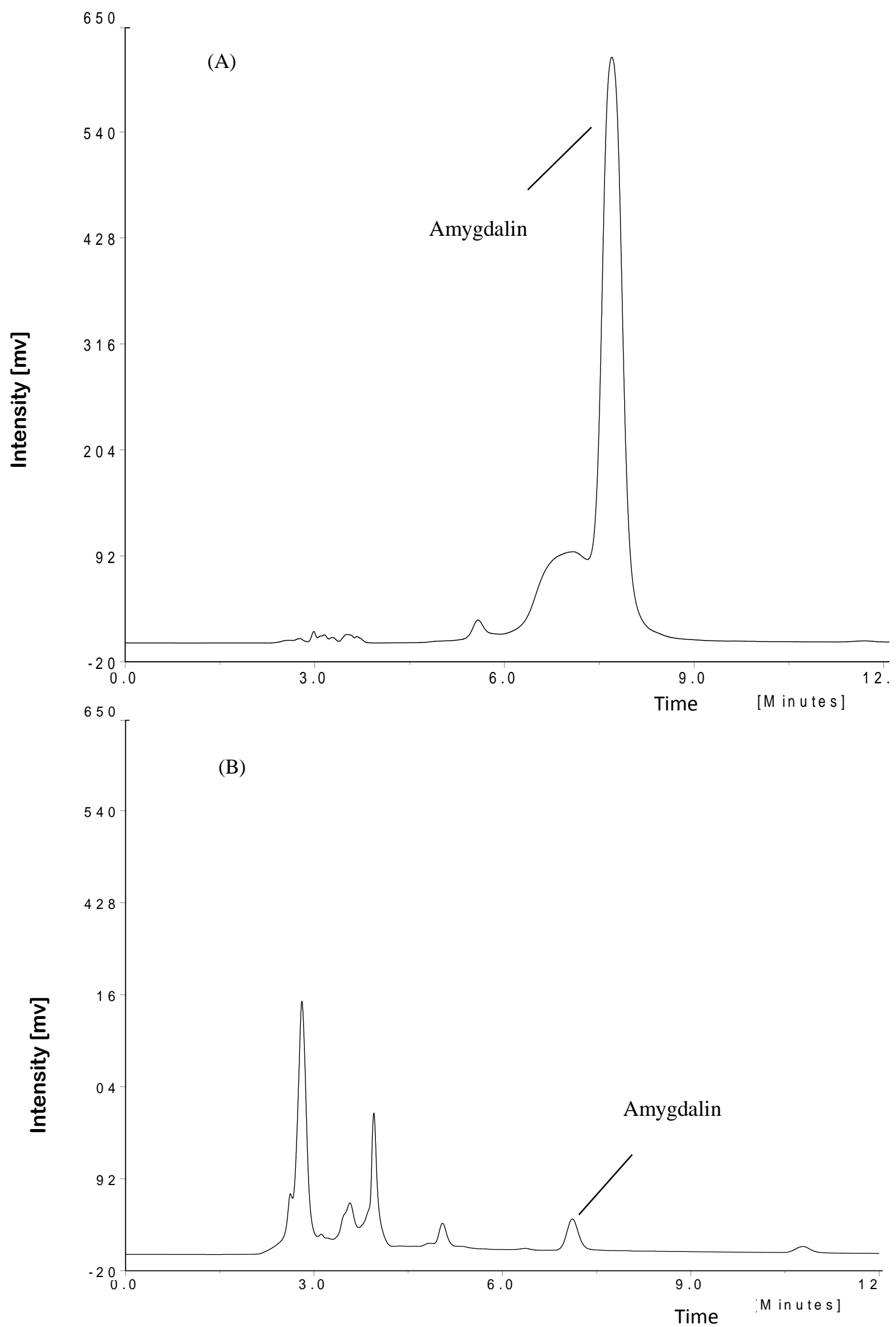

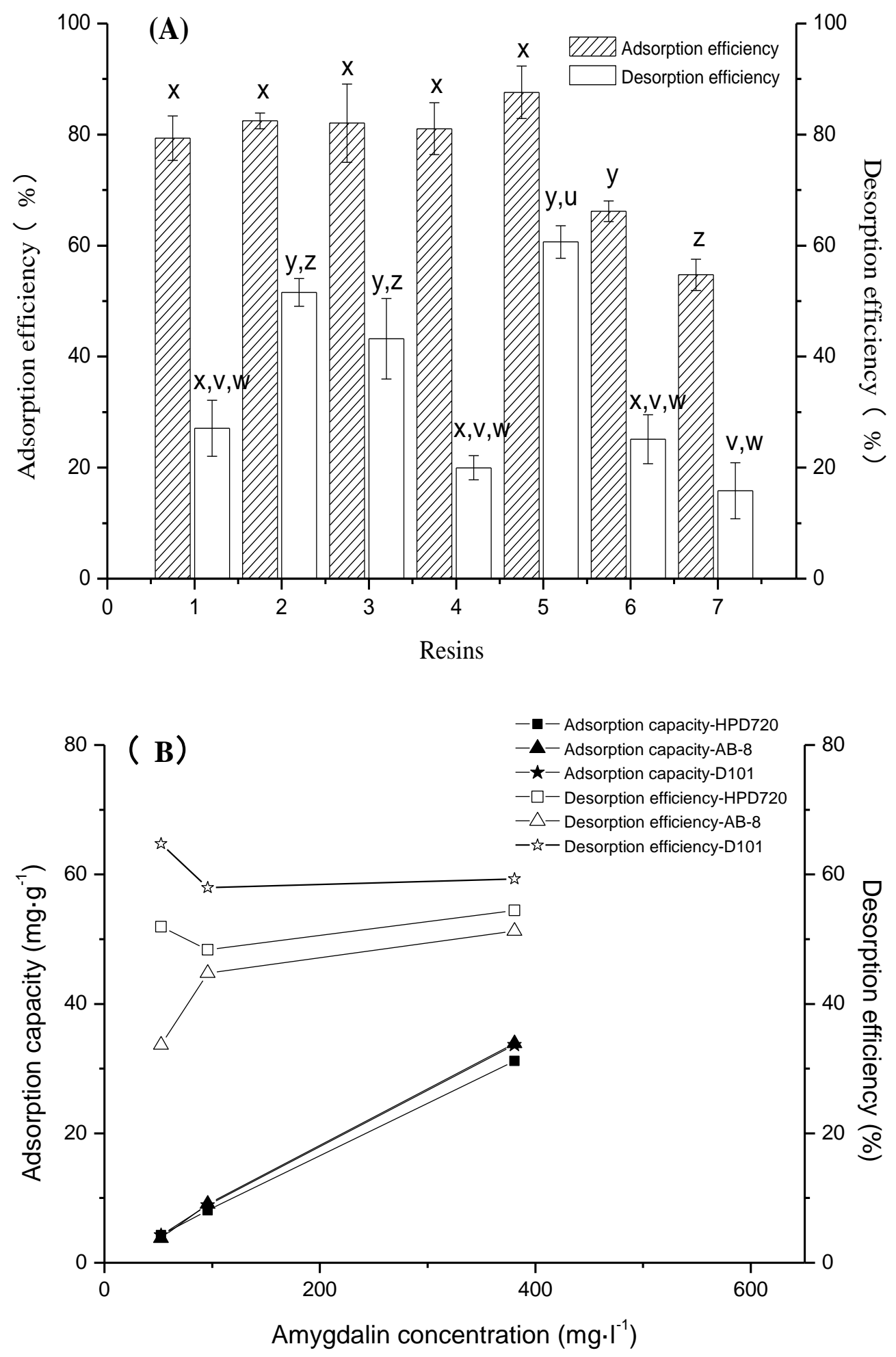
Fig.3 (A)- (G) T. Wang, et al.
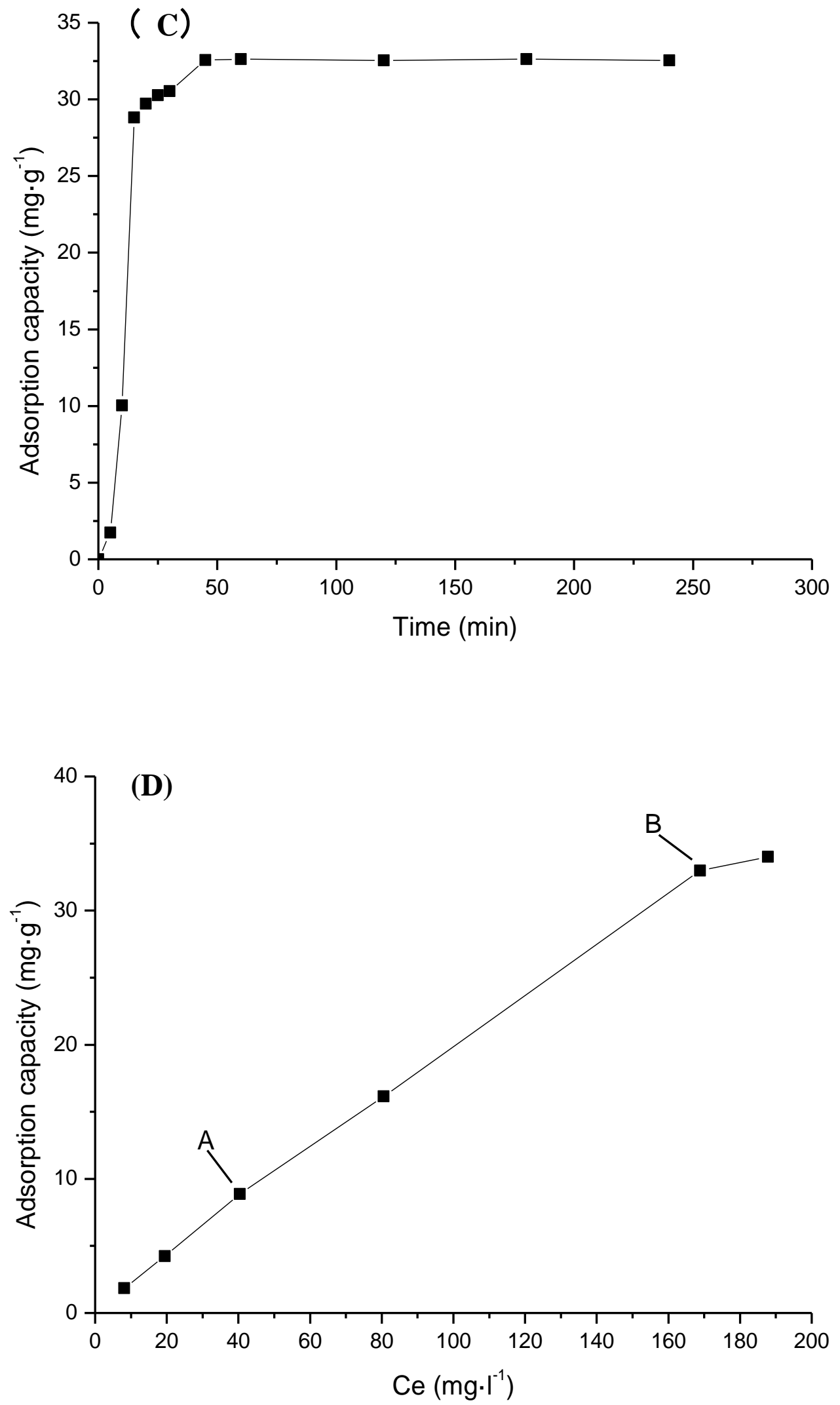
Fig.3 (A)- (G) T. Wang, et al.
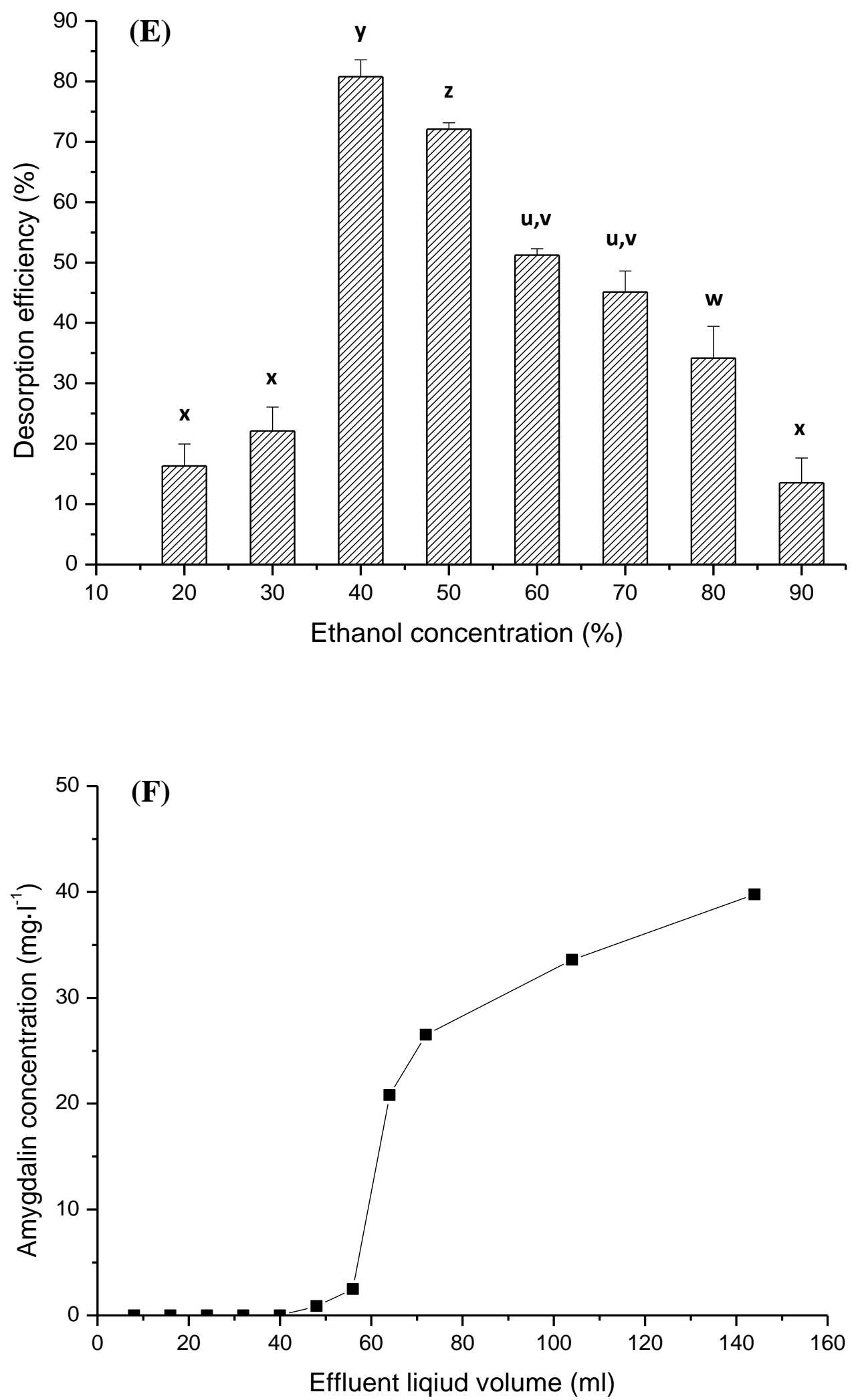


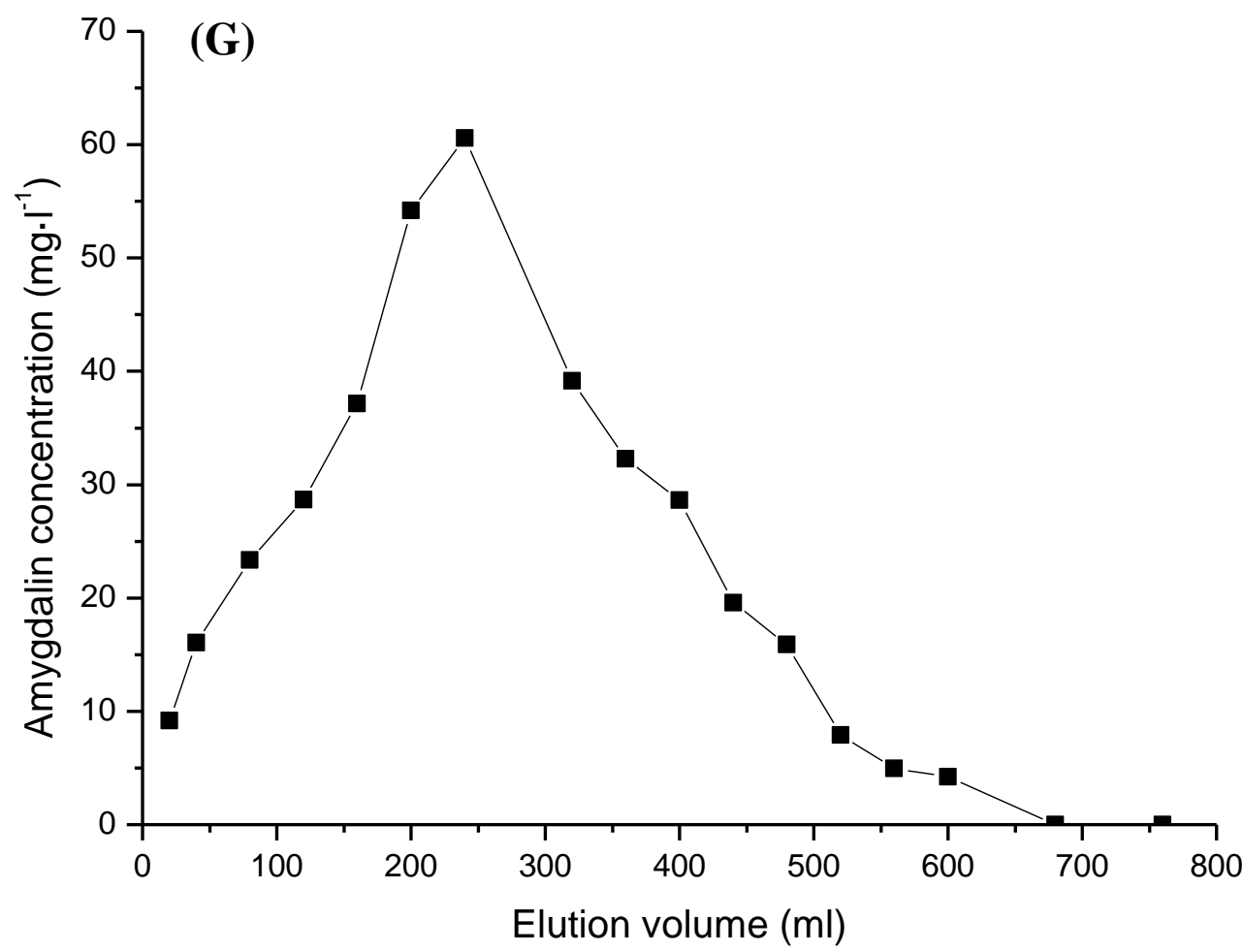


Fig.4 T. Wang, et al.

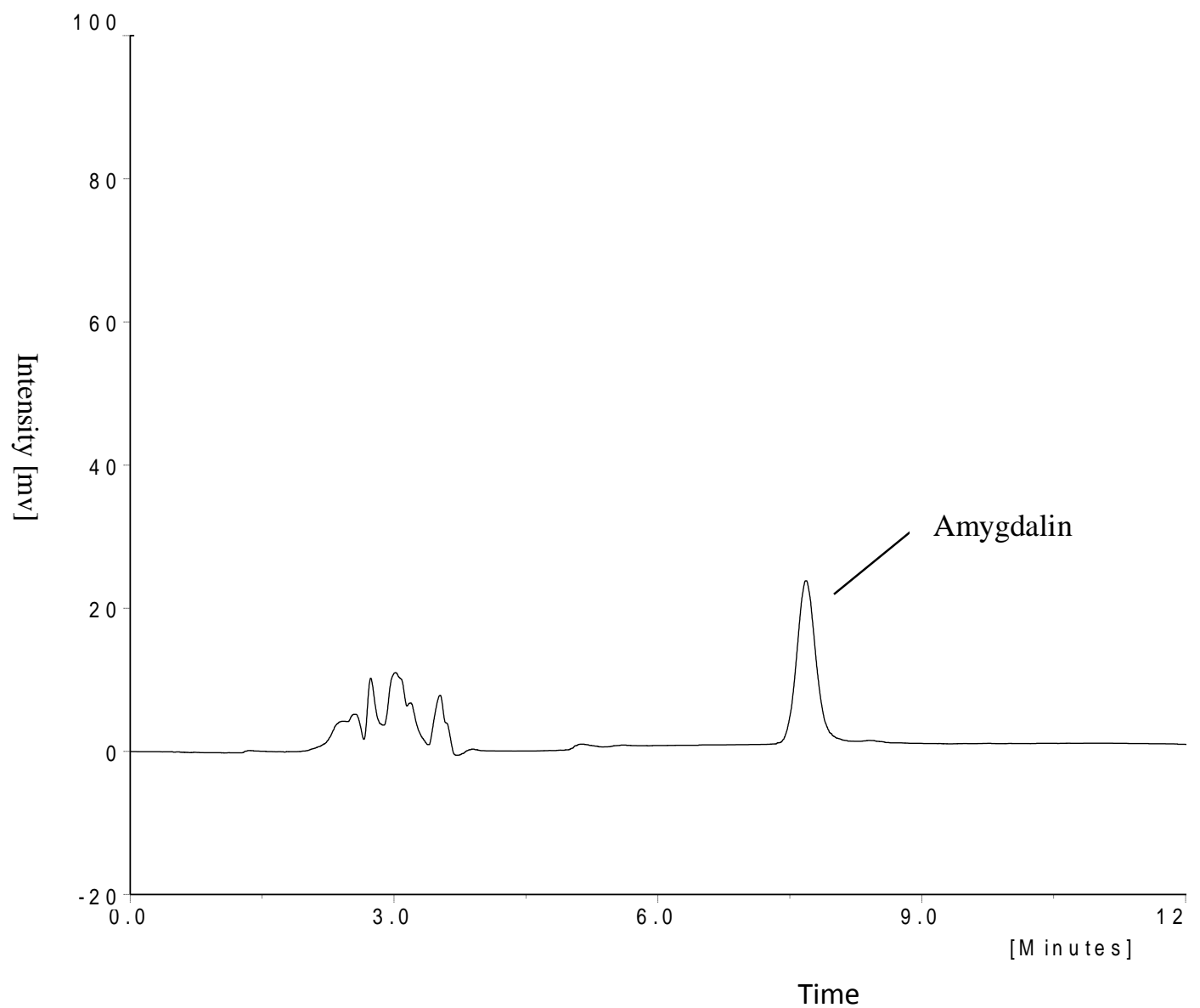

501 
503 Table 1 Physical properties and the moisture contents of the macroporous adsorption 504 resins

\begin{tabular}{cccccc}
\hline Resin & Polarity & Structure & Specific surface & Pore size & Moisture \\
\hline HPD722 & Weak & SDVB & $485-530$ & $13.0-14.0$ & 69.91 \\
HPD720 & Weak & SDVB & - & - & 67.52 \\
AB-8 & Weak & SDVB & $\geq 480$ & $13.0-14.0$ & 71.07 \\
NKA-9 & Weak & SDVB & $250-290$ & $15.5-16.5$ & 72.76 \\
D101 & Non & SDVB & $\geq 550$ & $9.0-10.0$ & 70.66 \\
HPD100 & Non & SDVB & $650-700$ & $8.5-9.0$ & 51.45 \\
HPD400 & Medium & SDVB & $500-550$ & $7.5-8.0$ & 68.69 \\
\hline
\end{tabular}

505 “_" : not specified. SDVB: styrene divinyl-benzene.

506 
507 Table 2 Langmuir and Freundlich parameters of amygdalin on D101 resin at $30^{\circ} \mathrm{C}$

\begin{tabular}{|c|c|}
\hline Linear equation & $C_{e} / q_{e}=4.4313+0.0052 C_{e}$ \\
\hline $\mathrm{R}^{2}$ & 0.9060 \\
\hline$q_{0}(\mathrm{mg} / \mathrm{g})$ & 192.3077 \\
\hline$K_{L}$ & 0.0012 \\
\hline
\end{tabular}

Freundlich

Linear equation

$\mathrm{R}^{2}$

$K_{F}$

$n$ $q_{e}=0.3080 C_{e}^{0.9040}$

0.9974

0.3080

1.1062

508 